\title{
On single limits and the asymptotic behaviour of separating turbulent boundary layers
}

\author{
DANIEL O. A. CRUZ and ATILA P. SILVA FREIRE ${ }^{\dagger}$ \\ Mechanical Engineering Department, Post-Graduate School of the Federal University of Rio de \\ Janeiro (COPPE/UFRJ), C.P. 68503, 21945-970, Rio de Janeiro, Brazil
}

(Received 25 October 1996 and in final form 12 November 1997)

\begin{abstract}
The present work studies the asymptotic structure of the turbulent boundary layer near a separation point through the single limit concept of Kaplun. A new scaling procedure is introduced resulting in a changeable asymptotic structure which is consistent with the experimental data. The classical structure of the velocity and temperature boundary layers is shown to develop into a one-deck structure near the separation joint due to the merging of the two principal equations. The Reynolds analogy breaks down yielding a different power-law for the temperature profile. New laws of the wall for both the velocity and temperature fields are derived. (C) 1998 Elsevier Science Ltd. All rights reserved.
\end{abstract}

\section{INTRODUCTION}

The description of a turbulent flow near a separation point is a very important but difficult problem. Indeed, the reduction to zero of the main scaling parameters in the asymptotic theory of turbulent boundary layer flow completely breaks down the classical two-deck structure. As a result, several analyses where alternative characteristic scaling parameters are proposed were developed over the last five years. These analyses explain how the classical former asymptotic developments for zero pressure gradients boundary layers can be modified such that a $y^{1 / 2}$-power law replaces the log law. Most works, however, are restricted to analyses of the velocity boundary layer problem only.

The purpose of this work is to study the asymptotic structure of both the velocity and the temperature turbulent boundary layers near a separation point. The flows of interest here are those that separate due to adverse pressure gradients in or on smoothly varying surfaces such as, for example, the flows through a diffuser or over a turbine blade. Unfortunately, there have been few studies on the heat transfer process of separating flows, most of which have dealt with flows that separate due to abrupt changes in geometry. The experimental data on heat transfer on separating flows show a drop on the heat transfer coefficient at separation followed by a sharp rise in the reattachment zone, and this behaviour must somehow be captured by any advanced theory. The implication is that, as the separation point is approached, the velocity and the temperature fields have different asymptotic structures, the Reynolds analogy breaks down and different power-law expressions emerge from the near wall equations.

† Author to whom correspondence should be addressed.
Here, we develop a detailed asymptotic analysis near a separation point to determine the local behaviour of the velocity and the temperature profiles, and consequently of the skin-friction coefficient and the Stanton number. From these results, any Reynolds analogy will then be possible to assess.

For the classical problem of zero-pressure-gradient boundary layers, early developments by Yajnik [1], Mellor [2] and Bush and Fendell [3] have stated the boundary layer to have a two-layered structure consisting of a viscous wall layer and an outer defect region. The two former authors do not use any closure hypothesis to represent the turbulent shear stress terms, relying on their analyses only on asymptotic reasoning. Bush and Fendell, on the other hand, use in their developments turbulence models of the mixinglength/eddy viscosity type. In all cases, the flow structure and solutions are developed in terms of an appropriate small parameter, $\varepsilon=\operatorname{ord}(\ln R)^{-1}$. Using a completely different asymptotic approach, Melnik [4] instead has claimed the boundary layer to have a three-deck structure. His formulation is based on a two-parameter expansion of the boundary layer equations, the new additional small parameter resulting from the particular turbulence closure model he uses. Sychev and Sychev [5] are also positive in saying that, even for flow without a pressure gradient, the boundary layer structure is three-layered.

In fact, all new discussions that have led to the development of three-layered asymptotic models for the turbulent boundary layer are motivated by the inability of the classical two-deck model to deal with large adverse pressure gradients. When a turbulent boundary layer is subjected to a large adverse pressure gradient, the wake velocity deficit is large and the mean momentum equation is non-linear. These features make Millikan's 'matchability' arguments, 


\section{NOMENCLATURE}

A constant in Stratford's law of the wall

$B$ constant in the temperature law of the wall

$c_{\mathrm{p}} \quad$ specific heat at constant pressure

$c_{\mu} \quad$ constant in $k-\varepsilon$-model

$C, b_{m}$ constants in the velocity law of the wall

$C_{\mathrm{f}} \quad$ skin friction coefficient

$D$ formal limit domain

$E$ denotes partial differential equation

$E_{1}, E_{2}$ parameter in law of the wall

$F, G$ generic functions defined in $\Xi$ by a system of differential equations

$H$ enthalpy

$k$ von Karman's constant

$K \quad$ turbulent kinetic energy

$L \quad$ characteristic length in viscous region

Pr Prandtl number

$p, P$ pressure

$q, Q$ heat flux

$R \quad$ Reynolds number

$S$ solutions of $E$

$S t \quad$ Stanton number

$t, T$ temperature

$t_{t} \quad$ friction temperature

$u, v \quad$ velocity components

$u_{\tau}$ friction velocity

$u_{R}$ reference velocity

$u^{+} \quad$ inner wall reference velocity $=u / u_{\tau}$

$x, y$ flow Cartesian coordinates

$y^{+} \quad$ inner wall reference scale $=y u_{\tau} / v$.
Greek symbols

$\alpha \quad$ overlap index defined by equation (17)

$\delta$ gauge function

$\Delta, \eta$ functions defined in $\Xi$

$\varepsilon \quad$ small parameter

$\theta \quad$ error function defined by equation (15)

$\mu \quad$ viscosity

$v \quad$ kinematic viscosity

$\Xi \quad$ space of all positive continuous functions on $(0,1]$

$\rho$ density

$\tau \quad$ shear stress.

\section{Subscripts}

1,2 order of magnitude

$d$ singularity point

$i, j \quad$ summation

1 local conditions

$\mathrm{p} \quad$ first grid point

$t$ temperature

w conditions at wall

$\Delta \quad$ variable stretched according to $\Delta(\varepsilon)$

$\eta \quad$ variable stretched according to $\eta(\varepsilon)$

$\infty \quad$ external flow conditions.

\section{Superscripts}

turbulent fluctuation

function transformed according to definition 1 . which result is a log-law and in a two deck structure, not valid anymore. Also the friction velocity, $u_{\tau}$, used in the classical approaches as a characteristic velocity, becomes an inappropriate scaling parameter for adverse pressure gradient boundary layers since it tends to zero. All these difficulties force into the adverse pressure gradient problem a new small parameter of the order of $R^{-1 / 3}$, which is used to scale a power $y$ layer that replaces Millikan's log-layer. This layer matches, on passage of the inner and outer limits, respectively, to the wall and defect layers. Thus, according to this picture, three sets of characteristic scales are needed for the asymptotic description of adverse pressure gradient turbulent boundary layers (see Durbin and Belcher [6]).

A major difficulty of all previous theories is to establish a single scaling procedure which can naturally accommodate the far upstream boundary layer structure to a two-deck structure, and the far downstream structure to a three-deck structure. In other words, the theories are not capable of explaining, in asymptotic terms, how the logarithmic layer vanishes as separation is approached, and how the $y^{1 / 2}$-layer is formed. In fact, some theories (Melnik [4], Mellor [7], Gersten [8]) present expressions for the intermediate layer that upon appropriate limit passages reduce to the log-law upstream and to the $y^{1 / 2}$-law downstream. These expressions, however, are developed in terms of inappropriate scaling parameters or conceptual frameworks that cannot explain how the logarithmic portion completely vanishes as $u_{\mathrm{r}} \rightarrow 0$.

In the present work a new scaling procedure is presented which is not subject to the aforementioned prejudices. A scaling parameter is defined through an algebraic equation, resulting in a changeable asymptotic structure for the boundary layer, different from those of other authors, but consistent with the experimental data. The flow structure is studied through the single limit process concept of Kaplun [9], together with his Ansatz about domains of validity. Thus, some formal properties of the motion equations, yielded by the definition of 'equivalent in the limit' of Kaplun, are used to determine the actual validity domains and overlap regions. The present work follows the approaches of Yajnik and of Mellor, not imposing any functional relationship between quantities determined 
by the Reynolds stress field and by the velocity field. These two fields are related only through some order of magnitude hypotheses about the velocity fluctuations.

A common fact is that a thermal turbulent boundary layer far upstream of a separation point also has a classical two-layered asymptotic structure, except that now the Prandtl number will be a parameter in the inner layer scaling. The Reynolds analogy holds and a temperature log-law exists. As separation is approached, however, we have just seen that the velocity boundary layer changes into a new asymptotic structure, and this somehow must also occur with the thermal boundary layer. The disappearance of the velocity log-law region must be reflected on the temperature field, resulting in the appearance of some temperature power- $y$ law. Here we show that, depending on the Prandtl number, as the separation is approached, the velocity and temperature asymptotic structures are different, the Reynolds analogy completely breaks down, and a different power-law emerges for the temperature profile. By means of an appropriate scaling, a new expression is derived for the temperature law of the wall which reduces to the log-law upstrean of the separation and to a powerlaw near the separation point. Extension of this expression to the defect layer by specification of a wake function will result in a Stanton number equation. The equation shows the Stanton number to pass through a finite value at the point of zero skin-friction.

The theory leads to a new expression for the law of the wall and to a skin-friction equation that holds up to the separation point. The latter equation can be constructed by consideration of a general defect law for adverse pressure gradient boundary layers. The results for the reverse flow region show that logarithmic profiles are also found there.

All theoretical results are validated with the backward-facing step flow data of Vogel and Eaton [10] and with the divergent channel flow data of Driver and Seegmiller [11]. In addition, a numerical implementation of the theory is made so as to compare the present formulation with more complex and expensive approaches. In particular, a comparison is made with the RSM formulation of Hwang and Peng [12]. The new results presented here include velocity, skin-friction and Stanton number profile predictions.

\section{KAPLUN LIMITS}

In this section, a survey of some essential ideas used in solving singular perturbation problems is made. Some of the concepts to be discussed here are those of matching of asymptotic expansions, domain of validity of such expansions, overlap, formal validity of equations and limit processes.

The purpose of perturbation methods is to try to construct approximating solutions by the study of simplified equations. For the class of problems termed singular perturbation problems, at least two expan- sions are needed to construct a solution which is uniformly valid in the whole interval domain. It is thus necessary to define the concept of uniform domain of validity for such approximations. This can be achieved by a direct extension of the concepts of uniform convergence on an interval and of uniform validity on an interval, to the concepts of uniform convergence on a function class and of uniform validity on a function class. The concepts of domain of validity, of overlap, of limit processes and of matching then follow immediately.

Matching is, by its nature, a comparison of two approximations in their domain of overlap. On this ground, rules and recipes can be devised for matching in which the concept of overlap does not appear explicitly. The well known technique of interchanging limit expansions can be shown to be appropriate for certain simpler cases. In simpler cases, even more precise rules can be enunciated such as the matching principle of Van Dyke [13]. This leads us to the central problem in perturbation theory: how can one justify a priori that two approximations have an overlap domain?

Trying to overcome this difficulty, Kaplun [9] suggested to consider some formal properties of equations, characterizing them through their domains of validity. This would be not only more basic for understanding the matching process but also essential in the construction of the asymptotic expansions. Since all techniques used for matching are based on overlap, it is clear that this can only be achieved if two approximations have a common validity domain. The formal properties of an approximation are defined through a study of limits of the original equation. Then, the concepts of formal domain, of intermediate equation, of principal equation and of formal domain of validity can be introduced. The operational details of the mathematical procedure are laid by five definitions, one Axiom and one Ansatz. These are shown below.

The formulation to be presented here is only introductory to the ample sets of results presented in Kaplun [9] and in Lagerstrom and Casten [14]. For more details on the technique, the reader is referred to these two works. Complementary material is found in Meyer [15], in Freund [16] and in Silva Freire and Hirata [17].

Here we use the topology on the collection of order classes as introduced by Meyer [15].

Let $\varepsilon$ be a parameter on $(0,1]$ and $x$ a variable in $R^{n}$ with Euclidean norm $|x|$. Let $F$ be a function defined for $\varepsilon$ and on some $x$-space domain with pointwise norm $\|F\|$. Our interest is to study the behaviour of $F$ in the limit $\varepsilon \rightarrow 0$. In particular, we are interested in the cases where singularities arise. For example, passage of the limit may result in the loss of the highest order derivative term in a differential equation, and hence in the impossibility of satisfying all the boundary conditions. The idea of the Kaplun limit is to study the limit as $\varepsilon \rightarrow 0$ not for fixed $x$ near a singu- 
larity point $x_{d}$, but for $x$ tending to $x_{d}$ in a definite relationship to $\varepsilon$ specified by a stretching function $\eta(\varepsilon)$.

Taking $x_{d}=0$, we define

$$
x_{\eta}=\frac{x}{\eta(\varepsilon)}, \quad G\left(x_{\eta} ; \varepsilon\right)=F(x ; \varepsilon)
$$

with $\eta(\varepsilon)$ a function defined in $\Xi$ (= space of all positive continuous functions on $(0,1])$.

The Kaplun limit process is then defined as follows.

Definition 1 (Meyer [15]). If the function

$$
G\left(x_{\eta} ;+0\right)=\lim _{\varepsilon \rightarrow 0} G\left(x_{\eta} ; \varepsilon\right)
$$

exists uniformly on $\left\{x /\left|x_{\eta}\right|>0\right\}$; then we define $\lim _{n} F(x ; \varepsilon)=G\left(x_{\eta},+0\right)$.

If $F$ is a function defined by a system of differential equations, then the above definition establishes to every order of $\eta$ a correspondence original equation $\stackrel{\lim _{n}}{\longrightarrow}$ associated equation on the subset of $\Xi$ for which the associated equation exists. The passage of the $\eta$ limit process is a formal operation which results in a set of associated equations referred to by Kaplun [9] as the 'splitting' of the original differential equation; this operation establishes the basis for the definition of formal domain of validity.

Definition 2. The formal limit domain of an associated equation $E$ is the set of orders $\varepsilon$ such that the $\eta$-limit process applied to the original equation yields $E$.

To evaluate how close two equations are, Kaplun needed to advance a measuring procedure. This was made through the definition of equivalent in the limit.

Definition 3. Two equations $E_{1}$ and $E_{2}$ are said to be equivalent in the limit for a given limit process, $\lim _{n}$, and to a given order $\delta(\varepsilon)$, if,

$$
\theta=\frac{E_{1}\left(x_{\eta} ; \varepsilon\right)-E_{2}\left(x_{\eta} ; \varepsilon\right)}{\delta(\varepsilon)} \rightarrow 0, \quad \text { as } \varepsilon \rightarrow 0 .
$$

Definition 4 (of formal domain of validity). The formal domain of validity to order $\delta(\varepsilon)$ of an equation $E$ of formal limit domain $D$ is the set $D_{e}=D \cup D_{i}^{\prime} \mathrm{s}$, where $D_{i}^{\prime}$ s are the formal limit domains of all equations $E_{i}$ such that $E$ and $E_{i}$ are equivalent to $D_{i}$ to order $\delta(\varepsilon)$.

To relate the formal domain of validity of an equation to its actual domain of validity, Kaplun [9] advanced two assertions, the Axiom of Existence and the Ansatz about domains of validity. These assertions are primitive and unverifiable assumptions of perturbation theory. They allow one to use definitions 14 to find approximate solutions to singular perturbation problems. Because the heuristic nature of the Axiom and of the Ansatz, comparison to experiments will always be important for validation purposes. The theory, however, as implemented through the above operations, is always very helpful in understanding the matching process and in constructing the appropriate asymptotic expansions.

Axiom (of Existence) (Kaplun [9]). If equations $E_{1}$ and
$E_{2}$ are equivalent in the limit to order $\delta(\varepsilon)$ for a certain region, then given a solution $S_{1}$ of $E_{1}$ which lies in the region of equivalence of $E_{1}$ and $E_{2}$, there exists a solution $S_{2}$ of $E_{2}$ such that as $\varepsilon \rightarrow 0,\left|S_{1}-S_{2}\right| / \delta \rightarrow 0$, in the region of equivalence of $E_{1}$ and $E_{2}$.

To this axiom, there corresponds an Ansatz to ensure that there exists a solution $S_{1}$ of $E_{1}$ which lies in the region of equivalence of $E_{1}$ and $E_{2}$.

Ansatz (about domains of validity) (Kaplun [9]). An equation with a given formal domain of validity $D$ has a solution whose actual domain of validity corresponds to $D$.

The word 'corresponds to' in the Ansatz was assumed by Kaplun to actually mean 'is equal to'. The above formulation ceases to be valid when small terms have large integrated effects. In the example to be studied here, however, the principle is expected to work. Switchback terms, which are deduced from inspection of formally higher order terms, can always be included in the original formulation if we backtrack to the lower order terms. Large integrated effects occur when singularities occur in the approximating functions; these are not expected to occur here.

\section{THE EQUATIONS OF MOTION}

We consider the problem of an incompressible twodimensional turbulent flow over a smooth surface in a prescribed pressure distribution. The time-averaged motion equations; i.e., the continuity equation, the Navier-Stokes equation and the energy equation, can be cast as

$$
\begin{gathered}
\frac{\partial u_{j}}{\partial x_{j}}=0 \\
u_{j} \frac{\partial u_{i}}{\partial x_{j}}=-\frac{\partial p}{\partial x_{j}}-\varepsilon^{2} \frac{\partial}{\partial x_{j}}\left(\overline{u_{j}^{\prime} u_{i}^{\prime}}\right)+\frac{1}{R} \frac{\partial^{2} u_{i}}{\partial x_{j}^{2}} \\
u_{j} \frac{\partial t}{\partial x_{j}}=-\varepsilon^{2} \frac{\partial}{\partial x_{j}}\left(\overline{u_{j}^{\prime} t^{\prime}}\right)+\frac{1}{\operatorname{Pr} R} \frac{\partial^{2} t}{\partial x_{j}^{2}}
\end{gathered}
$$

where the notation is classical. Thus $\left(x_{1}, x_{2}\right)=(x, y)$ stand for the coordinates, $\left(u_{1}, u_{2}\right)=(u, v)$ for the velocities, $p$ for pressure, $t$ for temperature and $R$ and $P r$ for the Reynolds and the Prandtl numbers, respectively. The dashes are used to indicate a fluctuating quantity. In the fluctuation terms an overbar is used to indicate a time-average.

All mean variables are referred to some characteristic quantity of the external flow. The velocity fluctuations, on the other hand, are referred to a characteristic velocity $u_{R}$, firstly introduced in ref. [1]. This characteristic velocity, fundamental for the determination of the inner layers solution is known to reduce upstream and downstream of a separation point, respectively, to $u_{\tau}$ and to $(v(\mathrm{~d} p / \mathrm{d} x) / \rho)^{1 / 3}$. It must then be defined so as to comply with this behaviour. In the next section we will show how this can be done.

The small parameter $\varepsilon$ is defined as 


$$
\varepsilon=\frac{u_{R}}{U_{\infty}} .
$$

The temperature fluctuation is considered to be of the order of the friction temperature $t_{\tau}$, here defined as

$$
t_{\mathrm{t}}=\frac{q}{\rho c_{\mathrm{p}} u_{R}}
$$

Note that the classical definition of $t_{\tau}$ based on the friction velocity breaks down near a separation point. Now, based on the adverse pressure gradient results of Orlando et al. [18], we make

$$
\operatorname{ord}\left(u_{i}^{\prime}\right)=\operatorname{ord}\left(t^{\prime}\right) \text {. }
$$

The above assumptions concerning the relative order of magnitude of the various fluctuation terms, which are crucial for our future developments, have become a well established result in the asymptotic theories for turbulent boundary layer flow. For incompressible flow, the basic experimental support for them stems from the works of the Stanford Heat and Mass Transfer Group. The results of Kistler [9] and of Kistler and Chen [20] provide experimental support for compressible flows.

\section{THE VELOCITY AND THE TEMPERATURE BOUNDARY LAYER ASYMPTOTIC STRUCTURE}

We write the asymptotic expansions for the flow parameters as

$$
\begin{gathered}
u(x, y)=u_{1}(x, y)+\varepsilon u_{2}(x, y) \\
v(x, y)=\frac{\eta}{\Delta}\left[v_{1}(x, y)+\varepsilon v_{2}(x, y)\right] \\
p(x, y)=p_{1}(x)+\varepsilon p_{2}(x, y) \\
t(x, y)=t_{1}(x)+\varepsilon t_{2}(x, y) \\
u_{i}^{\prime}(x, y)=\varepsilon u_{i 1}^{\prime}(x, y)+\varepsilon^{2} u_{i 2}^{\prime}(x, y) .
\end{gathered}
$$

To find the asymptotic structure of the boundary layer we consider the following stretching transformation

$$
x_{\Delta}=\frac{x}{\Delta(\varepsilon)}, \quad y_{\eta}=\frac{y}{\eta(\varepsilon)}, \quad \hat{u}_{i}\left(x_{\Delta}, y_{\eta}\right)=u_{i}(x, y) .
$$

with $\Delta(\varepsilon)$ and $\eta(\varepsilon)$ defined on $\Xi$.

Upon substitution of equation (13) into equation (3.2) and upon passage of the $\eta$-limit process onto the resulting equation we get (Cruz and Silva Freire [21]) : $x$-momentum equation:

$$
\begin{aligned}
& \operatorname{ord} \eta=\operatorname{ord} 1: \quad \hat{u}_{1} \frac{\partial \hat{u}_{1}}{\partial x_{\Delta}}+\hat{v}_{1} \frac{\partial \hat{u}_{1}}{\partial y_{\eta}}+\frac{\partial \hat{p}_{1}}{\partial x_{\Delta}}=0 \\
& \text { ord } \varepsilon^{2}<\operatorname{ord} \eta<\operatorname{ord~1:~} \quad \hat{u}_{1} \frac{\partial \hat{u}_{1}}{\partial x_{\Delta}}+\hat{v}_{1} \frac{\partial \hat{u}_{1}}{\partial y_{\eta}}+\frac{\partial \hat{p}_{1}}{\partial x_{\Delta}}=0
\end{aligned}
$$

$$
\operatorname{ord} \varepsilon^{2}=\operatorname{ord} \eta: \quad \hat{u}_{1} \frac{\partial \hat{u}_{1}}{\partial x_{\Delta}}+\hat{t}_{1} \frac{\partial \hat{u}_{1}}{\partial y_{\eta}}+\frac{\partial \hat{p}_{1}}{\partial x_{\Delta}}=-\frac{\partial \overline{\hat{u}_{1} \hat{v}_{1}^{\prime}}}{\partial y_{\eta}}
$$

$$
\begin{gathered}
\operatorname{ord}(1 / \varepsilon R)<\operatorname{ord} \eta<\operatorname{ord} \varepsilon^{2}: \frac{\partial \overline{\hat{u}_{1}^{\prime} \hat{v}_{1}^{\prime}}}{\partial y_{\eta}}=0 \\
\operatorname{ord}(1 / \varepsilon R)=\operatorname{ord} \eta:-\frac{\partial \overline{\hat{u}_{1}^{\prime} \hat{v}_{1}^{\prime}}}{\partial y_{\eta}}+\frac{\partial^{2} \hat{u}_{2}}{\partial y_{\eta}^{2}}=0 \\
\operatorname{ord} \eta<\operatorname{ord}(1 / \varepsilon R): \frac{\partial^{2} \hat{u}_{2}}{\partial y_{\eta}^{2}}=0
\end{gathered}
$$

$y$-momentum equation :

$$
\begin{gathered}
\operatorname{ord} \eta=\operatorname{ord~1:} \quad \hat{u}_{1} \frac{\partial \hat{v}_{1}}{\partial x_{\Delta}}+\hat{v}_{1} \frac{\partial \hat{v}_{1}}{\partial y_{\eta}}+\frac{\partial \hat{p}_{1}}{\partial y_{\Delta}}=0 \\
\operatorname{ord} \eta<\operatorname{ord} 1: \frac{\partial \hat{p}_{1}}{\partial y_{\eta}}=0 .
\end{gathered}
$$

The term $\hat{u}_{1}\left(x_{\Delta}, y_{n}\right)$ is missing from equations (14e) and (14f) since from the no-slip condition $\hat{u}_{1}=0$ near the wall.

Equations (14c) and (14e) are distinguished in two ways : (i) they are determined by specific choices of $\eta$, and (ii) they are 'richer' than the others in the sense that, application of the limit process to them yields some of the other equations, but neither of them can be obtained from passage of the limit process to any of the other equations. Thus, in the language of Kaplun, these equations are called principal equations. Principal equations are important since they are expected to be satisfied by the corresponding limits of the exact solution. We then make the following definition.

Definition 5 (of principal equation). An equation $E$ of formal limit domain $D$, is said to be principal to order $\delta$ if :

(i) one can find another equation $E^{\prime}$, of formal limit domain $D^{\prime}$, such that $E$ and $E^{\prime}$ are equivalent in $D^{\prime}$ to order $\delta$;

(ii) $E$ is not equivalent to order $\delta$ to any other equation in $D$.

A complete solution to the problem should then according to the Axiom of Existence and Kaplun's Ansatz, be obtained from the principal $x$-momentum and $y$-momentum equations located at points ord $\eta=1$, ord $\eta=$ ord $\varepsilon^{2}$ and ord $\eta=$ ord $(1 / \varepsilon R)$. The formal domains of validity of these equations cover the entire domain and overlap in a region determined according to definition 4.

To find the overlap region of equations (4.7c) and (4.7e), we must show these equations to have a common domain where they were equivalent; thus, we must use Kaplun's concept of equivalent in the limit, definition 4. A direct application of the definition to equations $(4.7 \mathrm{c})$ and $(4.7 \mathrm{e})$ yields 
$\theta=\frac{\frac{\hat{u}_{1}}{\Delta} \frac{\partial \hat{u}_{1}}{\partial x_{\Delta}}+\frac{\hat{v}_{1}}{\eta} \frac{\partial \hat{u}_{1}}{\partial y_{\eta}}+\frac{1}{\Delta} \frac{\mathrm{d} \hat{p}_{1}}{\mathrm{~d} x_{\Delta}}-\frac{1}{R \eta^{2}} \frac{\partial^{2} \hat{u}_{1}}{\partial y_{\eta}^{2}}}{\varepsilon^{\alpha}}$.

Noting that the leading order term in region $\operatorname{ord}(1 / \varepsilon R)<\operatorname{ord} \eta<\operatorname{ord} \varepsilon^{2}$ is the turbulent term, of $\operatorname{ord}\left(\varepsilon^{2} / \eta\right)$, we normalize the above equation to order unity to find

$$
\bar{\theta}=\frac{\eta}{\varepsilon^{2}} \theta .
$$

The overlap domain is the set of orders such that the $\eta$-limit process applied to $\bar{\theta}$ tends to zero for a given $\alpha$. Then since ord $\left(\partial / \partial_{y}\right)=\varepsilon$ and $\operatorname{ord}(\partial / \partial x)=1$, the formal overlap domain is given by

$D=\left\{\eta / \operatorname{ord}\left(\varepsilon^{1+\alpha} R\right)^{-1}<\operatorname{ord} \eta<\operatorname{ord}\left(\varepsilon^{2+\alpha} \Delta\right)\right\}$.

According to Kaplun's Ansatz about domains of validity, the approximate equations, equations (13c) and (13e), only overlap if set (16) is a non-empty set, that is, if

$$
0 \leqslant \alpha \leqslant-\frac{1}{2}\left(\frac{\ln R \Delta}{\ln \varepsilon}+3\right) .
$$

The implication is that the two-deck turbulent boundary layer structure given by the two principal equations, equations (13c) and (13e), provides approximate solutions which are accurate to the order of $\varepsilon^{\alpha_{\max }}$, where $\alpha_{\max }$ is the least upper bound of the interval (4.10). This fundamental result can only be reached through the application of Kaplun's concepts and ideas to the problem.

We conclude that the turbulent boundary layer has a two-deck structure very much like the one derived by Sychev and Sychev. This structure, however, must change as a separation point is approached. We shall see this next.

Equation (14f) together with the boundary condition

$$
\mu \frac{\partial u_{2}}{\partial y}=\tau_{w}
$$

shows that far away from the separation point $u_{R}=u_{\tau}$. Close to the separation point in the limit $\Delta \rightarrow 0$, however, $u_{\tau} \rightarrow 0$, so that an alternative value must be sought for $u_{R}$. The characteristic velocity $u_{R}$ will be determined here through some order of magnitude considerations.

At the bottom of the overlap region, a balance between the turbulent and viscous stresses occurs so that we may write

$$
\frac{\partial}{\partial y}\left(\overline{-\rho u_{1}^{\prime} v_{1}^{\prime}}\right)+\mu \frac{\partial^{2} u_{2}}{\partial y^{2}}=\frac{\partial p_{1}}{\partial x} .
$$

In this region, the characteristic length is given by $v / u_{R}$. Then, considering that the turbuient fluctuations are of the order of the reference velocity, $u_{R}$, and that the viscous term can be approximated by

$$
\operatorname{ord}\left(\mu \frac{\partial u_{2}}{\partial y}\right)=\operatorname{ord}\left(\tau_{\mathrm{w}}\right)
$$

it results from simple order of magnitude arguments that the characteristic velocity can be estimated from the algebraic equation

$$
u_{R}^{3}-\frac{\tau_{w}}{\rho} u_{R}-\frac{\nu}{\rho} \frac{\partial p}{\partial x}=0 .
$$

In the limit $\Delta \rightarrow 0$,

$$
u_{R} \rightarrow\left(\frac{v}{\rho} \frac{\partial p}{\partial x}\right)^{1 / 3}
$$

recovering the characteristic velocity for the near separation point region proposed by Stratford [22] and by Townsend [23].

The characteristic velocity $u_{R}$ is determined by the highest real root of (20).

The implication is that, close to the separation point, $\operatorname{ord}\left(\varepsilon^{2}\right)=\operatorname{ord}(1 / \varepsilon R)$, and the two 'rich' equations merge giving origin to a new structure. This merging provokes the disappearance of the log-region, reducing the flow structure to a wake region and a viscous region.

The flow structure then becomes:

$x$-momentum equation:

$\operatorname{ord} \Delta=\operatorname{ord} 1: \quad \hat{u}_{2} \frac{\partial \hat{u}_{2}}{\partial x_{\Delta}}+\hat{v}_{2} \frac{\partial \hat{u}_{2}}{\partial y_{\eta}}+\frac{\partial \hat{p}_{2}}{\partial x_{\Delta}}=0$
$\operatorname{ord} \varepsilon^{2}<\operatorname{ord} \Delta<\operatorname{ord} 1: \quad \hat{u}_{2} \frac{\partial \hat{u}_{2}}{\partial x_{\Delta}}+\hat{v}_{2} \frac{\partial \hat{u}_{2}}{\partial y_{\eta}}+\frac{\partial \hat{p}_{2}}{\partial x_{\Delta}}=0$

$$
\begin{array}{r}
\operatorname{ord} \varepsilon^{2}=\operatorname{ord} \Delta: \quad \hat{u}_{2} \frac{\partial \hat{u}_{2}}{\partial x_{\Delta}}+\hat{v}_{2} \frac{\partial \hat{u}_{2}}{\partial y_{\eta}}+\frac{\partial \hat{p}_{2}}{\partial x_{\Delta}} \\
=-\frac{\partial \overline{\hat{u}_{1}^{2}}}{\partial x_{\Delta}}-\frac{\partial \overline{\hat{u}_{1}^{\prime} \hat{v}_{1}^{\prime}}}{\partial y_{\eta}}+\frac{\partial^{2} \hat{u}_{2}}{\partial x_{\Delta}^{2}}+\frac{\partial^{2} \hat{u}_{2}}{\partial y_{n}^{2}} \\
\operatorname{ord} \Delta<\operatorname{ord} \varepsilon^{2}: \frac{\partial^{2} \hat{u}_{2}}{\partial x_{\Delta}^{2}}+\frac{\partial^{2} \hat{u}_{2}}{\partial y_{\eta}^{2}}=0
\end{array}
$$

$y$-momentum equation:

$\operatorname{ord} \Delta=\operatorname{ord} 1: \quad \hat{u}_{2} \frac{\partial \hat{v}_{2}}{\partial x_{\Delta}}+\hat{v}_{2} \frac{\partial \hat{v}_{2}}{\partial y_{\eta}}+\frac{\partial \hat{p}_{2}}{\partial y_{\eta}}=0$
$\operatorname{ord} \varepsilon^{2}<\operatorname{ord} 1<\operatorname{ord} \Delta: \quad \hat{u}_{2} \frac{\partial \hat{v}_{2}}{\partial x_{\Delta}}+\hat{v}_{2} \frac{\partial \hat{v}_{2}}{\partial y_{\eta}}+\frac{\partial \hat{p}_{2}}{\partial y_{\eta}}=0$

$$
\begin{aligned}
\operatorname{ord} \varepsilon^{2}=\operatorname{ord} \Delta: & \hat{u}_{2} \frac{\partial \hat{v}_{2}}{\partial x_{\Delta}}+\hat{v}_{2} \frac{\partial \hat{v}_{2}}{\partial y_{\eta}}+\frac{\partial \hat{p}_{2}}{\partial x_{\Delta}} \\
= & -\frac{\partial \overline{\hat{v}_{i}^{\prime 2}}}{\partial x_{\Delta}}-\frac{\partial \overline{\hat{u}_{1}^{\prime} \hat{v}_{1}^{\prime}}}{\partial y_{\eta}}+\frac{\partial^{2} \hat{v}_{2}}{\partial x_{\Delta}^{2}}+\frac{\partial^{2} \hat{v}_{2}}{\partial y_{\eta}^{2}} \\
\operatorname{ord} \Delta & <\operatorname{ord} \varepsilon^{2}: \frac{\partial^{2} \hat{v}_{2}}{\partial x_{\Delta}^{2}}+\frac{\partial^{2} \hat{v}_{2}}{\partial y_{\eta}^{2}}=0 .
\end{aligned}
$$


At this point it is of interest to note that in the region $(\Delta, \eta)=\left(\varepsilon^{2}, \varepsilon^{2}\right)$ the full Navier-Stokes averaged equation is recovered. The leading order equations for $\hat{u}_{1}$ together with the no-slip condition at the wall gives $\hat{u}_{1}=0$.

According to the above results, a global solution for the problem can only be obtained through equations (22c) and (22e). Unfortunately, these non-linear equations are of difficult solution, having the turbulent term yet to be defined.

To study the temperature boundary layer asymptotic structure we will use the same procedure as before. Application of the stretching transformation defined by equations (13) to the energy equation followed by passage of the $\eta$-limit process, yields :

$$
\begin{gathered}
\operatorname{ord} \eta=\operatorname{ord} 1: \quad \hat{u}_{1} \frac{\partial \hat{t}_{1}}{\partial x_{\Delta}}+\hat{v}_{1} \frac{\partial \hat{t}_{1}}{\partial y_{\eta}}=0 \\
\operatorname{ord} \varepsilon^{2}<\operatorname{ord} \eta<\operatorname{ord} 1: \hat{u}_{1} \frac{\partial \hat{t}_{1}}{\partial x_{\Delta}}+\hat{v}_{1} \frac{\partial \hat{t}_{1}}{\partial y_{\eta}}=0 \\
\operatorname{ord} \eta=\operatorname{ord} \varepsilon^{2}: \hat{u}_{1} \frac{\partial \hat{t}_{1}}{\partial x_{\Delta}}+\hat{v}_{1} \frac{\partial \hat{t}_{1}}{\partial y_{\eta}}=-\frac{\partial \hat{\hat{v}_{1}^{\prime} \hat{t}_{1}^{\prime}}}{\partial y_{\eta}} \\
\operatorname{ord}(1 / \varepsilon R \operatorname{Pr})<\operatorname{ord} \eta<\operatorname{ord} \varepsilon^{2}: \frac{\partial \hat{v}_{1}^{\prime} \hat{t}_{1}^{\prime}}{\partial y_{\eta}}=0 \\
\operatorname{ord} \eta=\operatorname{ord}(1 / \varepsilon R \operatorname{Pr}): \quad-\frac{\partial \hat{v}_{1}^{\prime} \hat{t}_{1}^{\prime}}{\partial y_{\eta}}-\frac{\partial^{2} \hat{t}_{2}}{\partial y_{\eta}^{2}}=0 \\
\quad \operatorname{ord} \eta<\operatorname{ord}(1 / \varepsilon R \operatorname{Pr}): \frac{\partial^{2} \hat{t}_{2}}{\partial y_{\eta}^{2}}=0
\end{gathered}
$$

The above equations imply that the temperature turbulent boundary layer has a two-layered structure, the two 'rich' equations being located at points $\operatorname{ord} \eta=\operatorname{ord} \varepsilon^{2}$ and $\operatorname{ord} \eta=\operatorname{ord}(1 / \varepsilon R P r)$ of the $\Xi$ space. The consequence is that the outer 'rich' equations for both the velocity and the temperature boundary layers are always located at the same point of $\Xi$. The location of the inner 'rich' equations, however, differs by a scale factor, the Prandtl number. Thus, if $\operatorname{Pr}=1$, the asymptotic structures of both the velocity and the temperature boundary layers will be identical, and logarithmic solutions will arise.

The overlap domain of equations (23c) and (23e) can be calculated just in the same way as the velocity field overlap domain was calculated (set 16). For this reason, the procedure will not be repeated here. We just point out to the reader that for the temperature case the overlap domain will depend on the Prandtl number.

Near to a separation point, however, the temperature boundary layer structure must change, much in the same way as the velocity boundary layer struc- ture changes. To study this change was pass the $\Delta$ limit process onto equations (23a)-(23f) to obtain :

$$
\begin{aligned}
& \operatorname{ord} \Delta=\operatorname{ord~1:} \quad \hat{u}_{2} \frac{\partial \hat{t}_{2}}{\partial x_{\Delta}}+\hat{v}_{2} \frac{\partial \hat{t}_{2}}{\partial y_{\eta}}=0 \\
& \operatorname{ord} \varepsilon^{2}<\operatorname{ord} \Delta<\operatorname{ord~1:} \quad \hat{u}_{2} \frac{\partial \hat{t}_{2}}{\partial x_{\Delta}}+\hat{v}_{2} \frac{\partial \hat{t}_{2}}{\partial y_{\eta}}=0
\end{aligned}
$$

ord $\varepsilon^{2}<\operatorname{ord} \Delta<$ ord 1 :

$$
\begin{array}{r}
\hat{u}_{2} \frac{\partial \hat{t}_{2}}{\partial x_{\Delta}}+\hat{v}_{2} \frac{\partial \hat{t}_{2}}{\partial y_{\eta}}=-\frac{\partial \overline{\hat{v}_{1} \hat{t}_{1}^{\prime}}}{\partial y_{n}}-\frac{\partial \overline{\hat{u}_{1}^{\prime} \hat{t}_{1}^{\prime}}}{\partial x_{\Delta}} \quad(24 \mathrm{c}) \\
\operatorname{ord}(1 / \varepsilon R \mathrm{Pr})<\operatorname{ord} \Delta<\operatorname{ord} \varepsilon^{2}: \frac{\partial \overline{\hat{v}_{1}^{\prime} \hat{t}_{1}^{\prime}}}{\partial y_{\eta}}+\frac{\partial \overline{\hat{u}_{1}^{\prime} \hat{t}_{1}^{\prime}}}{\partial x_{\Delta}}=0
\end{array}
$$

$\operatorname{ord}(1 / \varepsilon R P r)=\operatorname{ord} \Delta$

$$
\frac{\partial^{2} \hat{t}_{2}}{\partial y_{\eta}^{2}}+\frac{\partial^{2} \hat{t}_{2}}{\partial x_{\Delta}^{2}}-\frac{\partial \overline{\hat{\sigma}_{1}^{\prime} \hat{t}_{1}^{\prime}}}{\partial y_{\eta}}-\frac{\partial \overline{\hat{u}_{1}^{\prime} \hat{t}_{1}^{\prime}}}{\partial x_{\Delta}}=0
$$

ord $\Delta<\operatorname{ord}(1 / \varepsilon R \operatorname{Pr}): \frac{\partial^{2} \hat{t}_{2}}{\partial y_{\eta}^{2}}+\frac{\partial^{2} \hat{t}_{2}}{\partial x_{\Delta}^{2}}=0$.

The temperature leading order equation together with the boundary condition gives the solution $t_{1}=T_{w}$, where $T_{w}$ stands for wall temperature. According to the above equations, the temperature boundary layer will have three different asymptotic structures depending on the order of magnitude of the Prandtl number. If the Prandtl number is order unity, $\operatorname{ord}\left(\varepsilon^{2}\right)=\operatorname{ord}(1 / \varepsilon R P r)$ near a separation point and the two 'rich' equations (5.2c) and (5.2e) merge yielding a structure identical to the velocity boundary layer structure. If ord $(P r)>$ ord(1), the two 'rich' equations will remain defined in different points of the $\Xi$ space and the temperature two-layered structure will be preserved. In the third possibility, ord $(P r)<\operatorname{ord}(1)$, the merging of the two temperature 'rich' equations will occur prior to the merging of the velocity 'rich' equations. This 'premature' merging occurs at point $(\eta \Delta)=\left((R P r)^{-2 / 3},(R P r)^{-2 / 3}\right)$. The flow structure for both cases $\operatorname{Pr}<1$ and $\operatorname{Pr}>1$ are shown in Fig. 1 .

These figures confirm all results that were expected to occur beforehand. For $\operatorname{Pr}<1$, the disappearance of the temperature log-region takes place before the disappearance of the velocity log-region. The converse is true for $\operatorname{Pr}>1$. The case $\operatorname{Pr}<1$ should then be more amenable to analytical treatment.

\section{LAW OF THE WALL FORMULATION}

Consider the normal velocity gradient in the fully turbulent region, or, alternatively, in a region just outside the viscous layer. According to the preceding section, the functional dependence of $\partial u / \partial y$ on the flow parameters must be of the form 


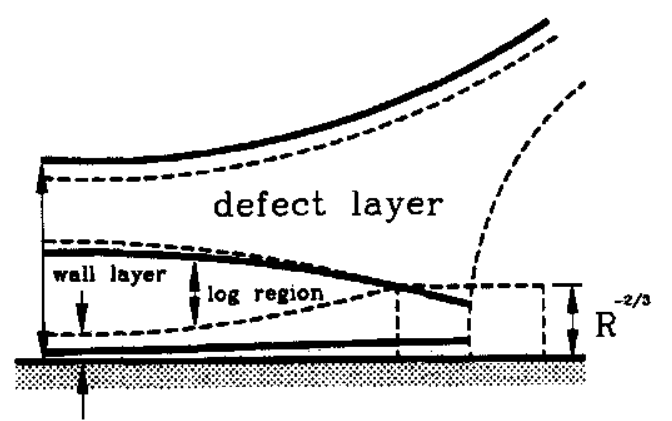

a) $P_{r}>1$

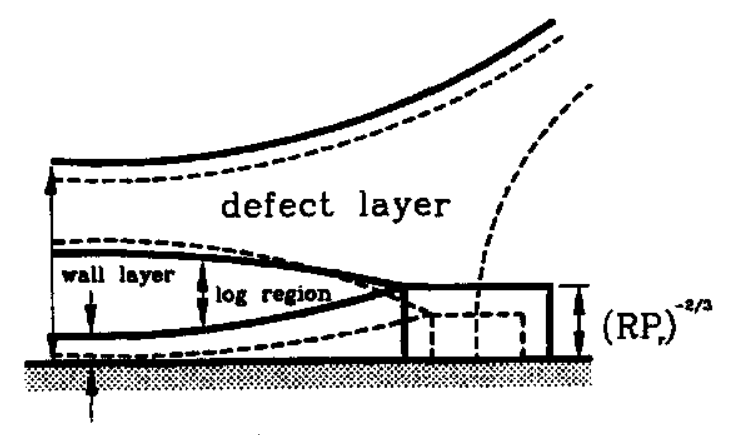

b) $\mathrm{P}_{r}<1$

Fig. 1. Asymptotic structure of the flow. -.-, velocity structure; -, temperature structure.

$$
\frac{\partial u}{\partial y}=f(y, \rho, \tau)
$$

where all symbols have their classical meaning. The above equation is not new, having been arrived at by simple dimensional considerations. (See, for example, Gersten [8], Landau and Lifshitz [24] and Kader and Yaglom [25].) The only combination of parameters $y$, $\rho, \tau$ which yields the dimension of $\partial u / \partial y$ is

$$
\frac{\partial u}{\partial y}= \pm \frac{1}{k} \sqrt{\frac{|z / \rho|}{y}}
$$

where $k$ is a constant (the Von Karman constant), and the sign + or - must agree with the direction of $\tau$. To solve this equation some consideration will have to be made about the behaviour of $\tau$. A Taylor series expansion around $y=0$, gives

$$
\tau=\tau_{w}+\frac{\mathrm{d} P_{\mathrm{w}}}{\mathrm{d} x} y+O\left(y^{2}\right)
$$

where $\tau_{\mathrm{w}}$ and $\mathrm{d} P_{\mathrm{w}} / \mathrm{d} x$ are, respectively, the shear stresses and the pressure at the wall. Substitution of the above equation into the previous one will give three different integration functions depending on the relative values of $\tau_{\mathrm{w}}$ and of $\left(\mathrm{d} P_{\mathrm{w}} / \mathrm{d} x\right)$.
Case $1:\left(\tau_{\mathrm{w}} / \rho\right) \geqslant 0 \rightarrow$

$$
\begin{aligned}
u= & \frac{2}{k} \sqrt{\tau_{\mathrm{w}} / \rho+\frac{1}{\rho} \frac{\mathrm{d} P_{\mathrm{w}}}{\mathrm{d} x} y}+\frac{\sqrt{\tau_{\mathrm{w}} / \rho}}{k} \\
& \times \ln \left|\frac{\sqrt{\tau_{\mathrm{w}} / \rho+\frac{1}{\rho} \frac{\mathrm{d} P_{\mathrm{w}}}{\mathrm{d} x}} y-\sqrt{\tau_{\mathrm{w}} / \rho}}{\sqrt{\tau_{\mathrm{w}} / \rho+\frac{1}{\rho} \frac{\mathrm{d} P_{\mathrm{w}}}{\mathrm{d} x} y}+\sqrt{\tau_{\mathrm{w}} / \rho}}\right|+C_{1}(x) .
\end{aligned}
$$

Case $2:\left(\tau_{\mathrm{w}} / \rho\right) \leqslant 0$ and $\left|(1 / \rho)\left(\mathrm{d} P_{\mathrm{w}} / \mathrm{d} x\right) y\right| \geqslant\left|\tau_{\mathrm{w}} / \rho\right| \rightarrow$

$$
\begin{aligned}
u=\frac{2}{k} \sqrt{\tau_{\mathrm{w}} / \rho} & +\frac{1}{\rho} \frac{\mathrm{d} P_{\mathrm{w}}}{\mathrm{d} x} y+\frac{\tau_{\mathrm{w}} / \rho}{\sqrt{-\tau_{\mathrm{w}} / \rho}} \frac{2}{k} \\
& \times \arctan \sqrt{\frac{\tau_{\mathrm{w}} / \rho+\frac{1}{\rho} \frac{\mathrm{d} P_{\mathrm{w}}}{\mathrm{d} x} y}{-\tau_{\mathrm{w}} / \rho}}+C_{2}(x) .
\end{aligned}
$$

Case $3:\left(\tau_{\mathrm{w}} / \rho\right) \leqslant 0$ and $\left|(1 / \rho)\left(\mathrm{d} P_{\mathrm{w}} / \mathrm{d} x\right) y\right| \leqslant\left|\tau_{\mathrm{w}} / \rho\right| \rightarrow$

$$
\begin{aligned}
u= & -\frac{2}{k} \sqrt{-\tau_{\mathrm{w}} / \rho-\frac{1}{\rho} \frac{\mathrm{d} P_{\mathrm{w}}}{\mathrm{d} x} y}-\frac{\sqrt{-\tau_{\mathrm{w}} / \rho}}{k} \\
& \times \ln \left|\frac{\sqrt{-\tau_{\mathrm{w}} / \rho-\frac{1}{\rho} \frac{\mathrm{d} P_{\mathrm{w}}}{\mathrm{d} x} y-\sqrt{-\tau_{\mathrm{w}} / \rho}}}{\sqrt{-\tau_{\mathrm{w}} / \rho-\frac{1}{\rho} \frac{\mathrm{d} P_{\mathrm{w}}}{\mathrm{d} x} y}+\sqrt{-\tau_{\mathrm{w}} / \rho}}\right|+C_{3}(x) .
\end{aligned}
$$

Equations (25)-(27) are a generalization of the classical law of the wall for separating flows. Far upstream from the separation point, $\tau_{\mathrm{w}} / \rho \gg$ $\left(\mathrm{d} P_{\mathrm{w}} / \mathrm{d} x\right)(y / \rho)$ and equation $(25)$ reduces to

$$
u^{+}=\frac{1}{k} \ln y^{+}+b_{m}
$$

the classical log-law.

Near to a separation point, equation (25) tends to

$$
u^{+}=\frac{2}{\beta k}\left(y^{+}\right)^{1 / 2}-A
$$

the Stratford's law of the wall.

Downstream of the separation point, in the region of reverse flow, equation (27) holds. Equally to equation (25), this equation has a logarithmic behaviour. The reverse flow direction, however, is immediately implied by the minus sign. Several experimental works have observed a logarithmic law in the reverse flow region (Simpson et al. [26], Thompson and Whitelaw [27], Dengel and Fernholtz [28]). We now turn to another very important point of our problem: the characteristic length scales of flow.

To find the viscous layer characteristic length, we again resort to the results of the previous section of the paper to write

$$
v \frac{\partial^{2} u}{\partial y^{2}}-\frac{1}{\rho} \frac{\mathrm{d} P_{\mathrm{w}}}{\mathrm{d} x}=0
$$


the approximate near wall flow motion equation. Successive integration of the above equation together with the boundary conditions at the wall yields

$$
u^{*} u_{\mathrm{\tau}}=\frac{\tau_{\mathrm{w}}}{\rho} \frac{L}{v} y^{*}+\frac{1}{\rho} \frac{\mathrm{d} P_{\mathrm{w}}}{\mathrm{d} x} \frac{L^{2}}{2 v} y^{* 2}
$$

where $u^{*}=u / u_{\tau}, y^{*}=y / L$ and $L$ is a length scale still to be determined. Considering that in the viscous region ord $\left(u^{*}\right)=\operatorname{ord}\left(y^{*}\right)=\operatorname{ord}(1)$, and passing the limit as $u_{\tau} \rightarrow 0$, we obtain

$$
L=\frac{-w_{\tau}^{2} \pm\left|-u_{\tau}^{2}\right|\left(1+\frac{v}{\tau_{\mathrm{w}}^{2}} \frac{\mathrm{d} P_{\mathrm{w}}}{\mathrm{d} x} u_{\tau}\right)}{\frac{1}{\rho} \frac{\mathrm{d} P_{\mathrm{w}}}{\mathrm{d} x}} .
$$

From the above equation, it results that

$$
L=\frac{2\left|u_{\tau}^{2}\right|}{\frac{1}{\rho} \frac{\mathrm{d} P_{\mathrm{w}}}{\mathrm{d} x}}
$$

As can be seen, the behaviour of $L$ downstream of the separation point is completely different from its upstream behaviour where $L=v / u_{\tau}$.

Similarly to the velocity field analysis, we start our investigation by looking at the near wall conduction region. Then, just outside of it, in the fully turbulent region, the flux of heat must have a functional dependence of the form

$$
\frac{\partial T}{\partial y}=g\left(y, \rho, c_{p}, q_{w}, \partial u / \partial y\right)
$$

where $T=T_{1}-T_{\text {w }}$ and $T_{1}$ and $T_{w}$ are, respectively, the local fluid temperature and the wall temperature.

$c_{\mathrm{p}}$ and $q_{\mathrm{w}}$ stand for the specific heat at a constant pressure and for the local flux of heat at the wall.

After dimensional considerations, we write

$$
\frac{\partial T}{\partial y}=B \frac{q_{\mathrm{w}}}{\rho c_{\mathrm{p}}} \frac{1}{\left(y^{2} \partial u / \partial y\right)}
$$

with $B$ a constant still to be determined. These equations will lead, again, to three different integration functions depending on the relative values of $\tau_{w}$ and of $\left(\mathrm{d} P_{\mathrm{w}} / \mathrm{d} x\right)$

Case $1:\left(\tau_{\mathrm{w}} / \rho\right) \geqslant 0 \rightarrow$

$$
\begin{aligned}
& T=\frac{1}{k_{t}} \frac{q_{\mathrm{w}}}{\rho c_{\mathrm{p}}} \frac{1}{\sqrt{\frac{\tau_{\mathrm{w}}}{\rho}}} \\
& \quad \times \ln \left|\frac{\sqrt{\tau_{\mathrm{w}} / \rho+\frac{1}{\rho} \frac{\mathrm{d} P_{\mathrm{w}}}{\mathrm{d} x} y}-\sqrt{\tau_{\mathrm{w}} / \rho}}{\sqrt{\tau_{\mathrm{w}} / \rho+\frac{1}{\rho} \frac{\mathrm{d} P_{\mathrm{w}}}{\mathrm{d} x}} y+\sqrt{\tau_{\mathrm{w}} / \rho}}\right|+C_{t 1}(x, P r) .
\end{aligned}
$$

Case $2:\left(\tau_{\mathrm{w}} / \rho\right) \leqslant 0$ and $\left|(1 / \rho)\left(\mathrm{d} P_{\mathrm{w}} / \mathrm{d} x\right) y\right| \geqslant\left|\tau_{\mathrm{w}} / \rho\right| \rightarrow$

$$
T=\frac{1}{k_{t}} \frac{g_{\mathrm{w}}}{\rho c_{\mathrm{p}}} \frac{2}{\sqrt{\frac{\tau_{\mathrm{w}}}{\rho}}} \arctan \sqrt{\frac{\tau_{\mathrm{w}} / \rho+\frac{1}{\rho} \frac{\mathrm{d} P_{\mathrm{w}}}{\mathrm{d} x} y}{-\tau_{\mathrm{w}} / \rho}}+C_{t 2}(x, P r)
$$

Case 3: $\left(\tau_{\mathrm{w}} / \rho\right) \leqslant 0$ and $\left|(1 / \rho)\left(\mathrm{d} P_{\mathrm{w}} / \mathrm{d} x\right) y\right| \leqslant \tau_{\mathrm{w}} / \rho \mid \rightarrow$

$$
\begin{array}{r}
T=\frac{1}{k_{t}} \frac{q_{\mathrm{w}}}{\rho c_{\mathrm{p}}} \frac{1}{\sqrt{\frac{-\tau_{\mathrm{w}}}{\rho}}} \\
\times \ln \left|\frac{\sqrt{-\tau_{\mathrm{w}} / \rho-\frac{1}{\rho} \frac{\mathrm{d} P_{\mathrm{w}}}{\mathrm{d} x} y}-\sqrt{-\tau_{\mathrm{w}} / \rho}}{\sqrt{-\tau_{\mathrm{w}} / \rho-\frac{1}{\rho} \frac{\mathrm{d} P_{\mathrm{w}}}{\mathrm{d} x} y}+\sqrt{-\tau_{\mathrm{w}} / \rho}}\right| \\
\end{array}
$$

These equations are the law of the wall for the temperature boundary layer in separating flows. Likewise the velocity expressions, these equations have the expected behaviour near and far away from the separation point. In fact, for the limit case, $\left|\tau_{\mathrm{w}} / \rho\right| \gg\left|\left(\mathrm{d} p_{\mathrm{w}} / \mathrm{d} x\right)(y / \rho)\right|$, equation (28) reduces to the classical law of the wall logarithmic profile given by

$$
\frac{t-t_{\mathrm{w}}}{t_{\tau}}=\frac{1}{k_{t}} \ln y^{+}+C_{\imath}(P r) .
$$

In the limit case $\tau_{w} \rightarrow 0$, however, equations (28)(30) reduce to expressions with the form

$$
\frac{t-t_{\mathrm{w}}}{t_{\tau}}=\frac{1}{k_{\mathrm{t}}} \frac{q_{\mathrm{w}}}{\rho c_{\mathrm{p}}} \frac{-2}{\sqrt{\frac{1}{\rho} \frac{\mathrm{d} P_{\mathrm{w}}}{\mathrm{d} x} y}}+C_{t 1}(x, \operatorname{Pr}) .
$$

This equation has a functional form different from Stratford's law of the wall, and this clearly characterizes the break down of the Reynolds analogy near a separation point. This fact has been experimentally observed by, for example, Vogel and Eaton [10]. The region of validity of equations (25)-(30) is illustrated in Fig. 2.

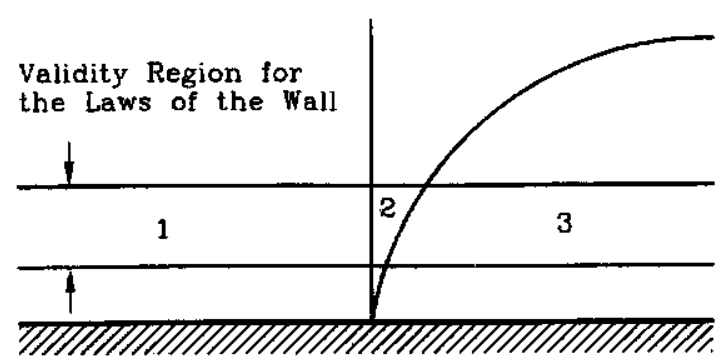

Fig. 2. Validity domain of laws of the wall. The separation point is located at the foot of region 2 . 
In what follows, we will give experimental evidence of the above formulation. This will be made in two ways : (1) by a direct analysis of the experimental data and the realization that the temperature profile has a minus-half-power behaviour, and (2) by a numerical implementation of expressions (25)-(30) in a finite volume method computer code. The predicted skinfriction, Stanton number and velocity profiles will then be compared with the experimental data.

\section{EXPERIMENTAL AND NUMERICAL VALIDATION}

The theoretical results will be validated with the backward-facing step flow data of Vogel and Eaton [10] and with the divergent channel flow data of Driver and Seegmiller [11]. The experimental flow conditions for both experiments are shown in Table 1.

The temperature profiles in the reverse flow regions are shown in Fig. 3. The occurrence of a logarithmic solution is evident as predicted by solution (30). In Fig. 4, the temperature profile at the separation point is shown. The existence of a flow region where $T_{\infty}-T \sim y^{-(1 / 2)}$ is apparent. The existence of an adjacent to the wall viscous sub-layer and of a defect layer

Table 1. Experiental flow conditions

\begin{tabular}{lccc}
\hline Author & $U\left[\mathrm{~m} \mathrm{~s}^{-1}\right]$ & $R$ & $Q_{\mathrm{w}}\left[\mathrm{W} \mathrm{m}^{-2}\right]$ \\
\hline Vogel and Eaton & 11.3 & 28000 & 270 \\
Driver and Seegmiller & 44.3 & 37000 & 0.0 \\
\hline
\end{tabular}

can also be seen in this figure. The half-power law for the velocity profile is shown in Fig. 5 . According with the theoretical results, this figure shows that the halfpower law holds not only at the separation point but also for the reverse flow region as predicted by equation (27).

A numerical implementation of the present theory was made with the help of the computer code CAST (Computer Aided Simulation of Turbulence, Peric and Scheuerer [29]). This program is similar in structure to other existing fluid flow prediction schemes such as TEAM and TEACH. It is thus a conservative finite-volume method in primitive variables. Differences from those codes arise in the co-located variable arrangement, the discretization scheme, the solution agorithms for the linear equation systems resulting form the discretization and in the pressure coupling which is adopted to the co-located variable storage.

For turbulent flow, the code solves the Reynolds averaged Navier-Stokes equations in connection with the $K-\varepsilon$ differential turbulence model of Launder and Spalding [30]. The five empirical constants appearing in the code take on the standard values. Since CAST uses the wall function method, updating the program was a relatively straightforward affair. To evaluate the momentum balance at the adjacent to the wall volume control, we made

to $\tau_{\mathrm{w}} \geqslant 0$ :

$$
\tau_{\mathrm{w}}=\kappa c_{\mu}^{1 / 4} \frac{\rho \sqrt{K_{\mathrm{p}}}}{\ln E_{1} y_{1}} u_{\mathrm{p}}
$$

to $\tau_{w}<0$ :

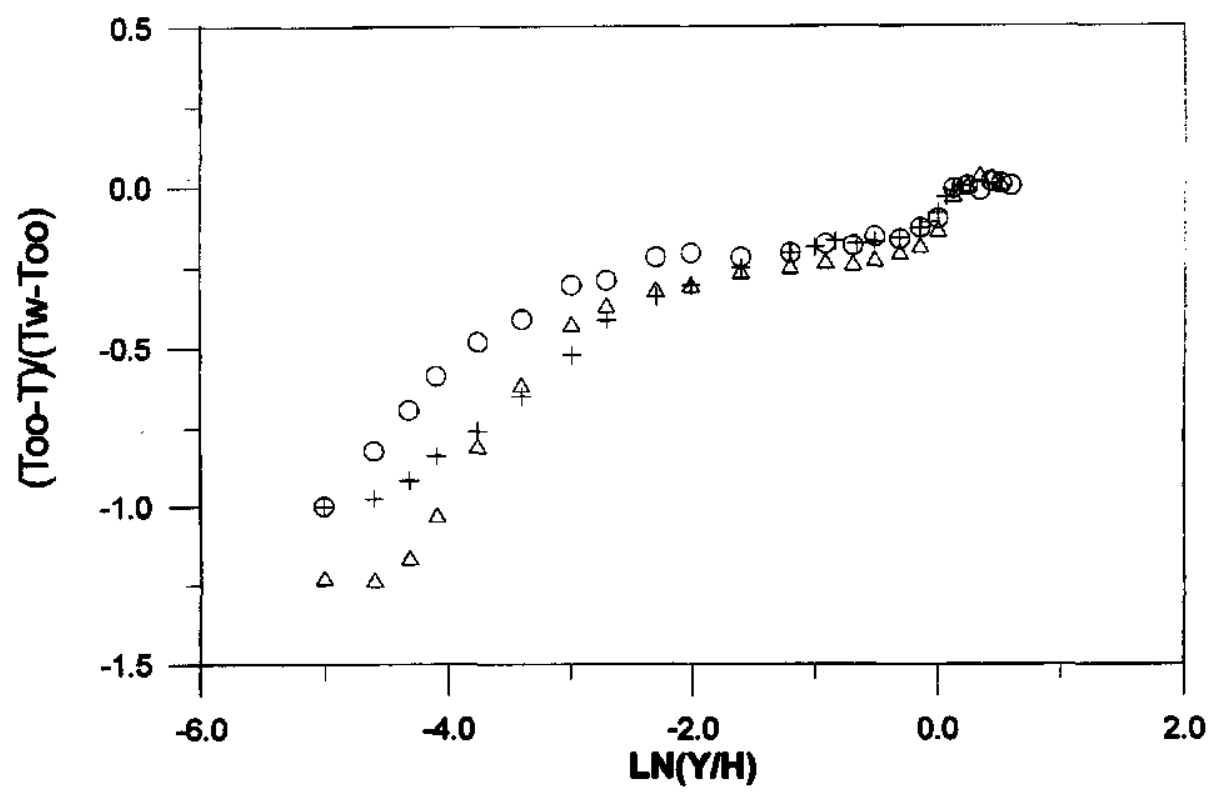

Fig. 3. Temperature profiles in reverse flow region according to Vogel and Eaton [10]. $y$ is referred to the step height, $H(=3.79 \mathrm{~cm})$. The points were taken at stations $x^{*}=-0.35(0),-0.55(\Delta)$ and -0.75 $(+)$, where $x^{*}=\left(x-x_{R}\right) / x_{R}, x_{R}=6.6643 \mathrm{H}$. 


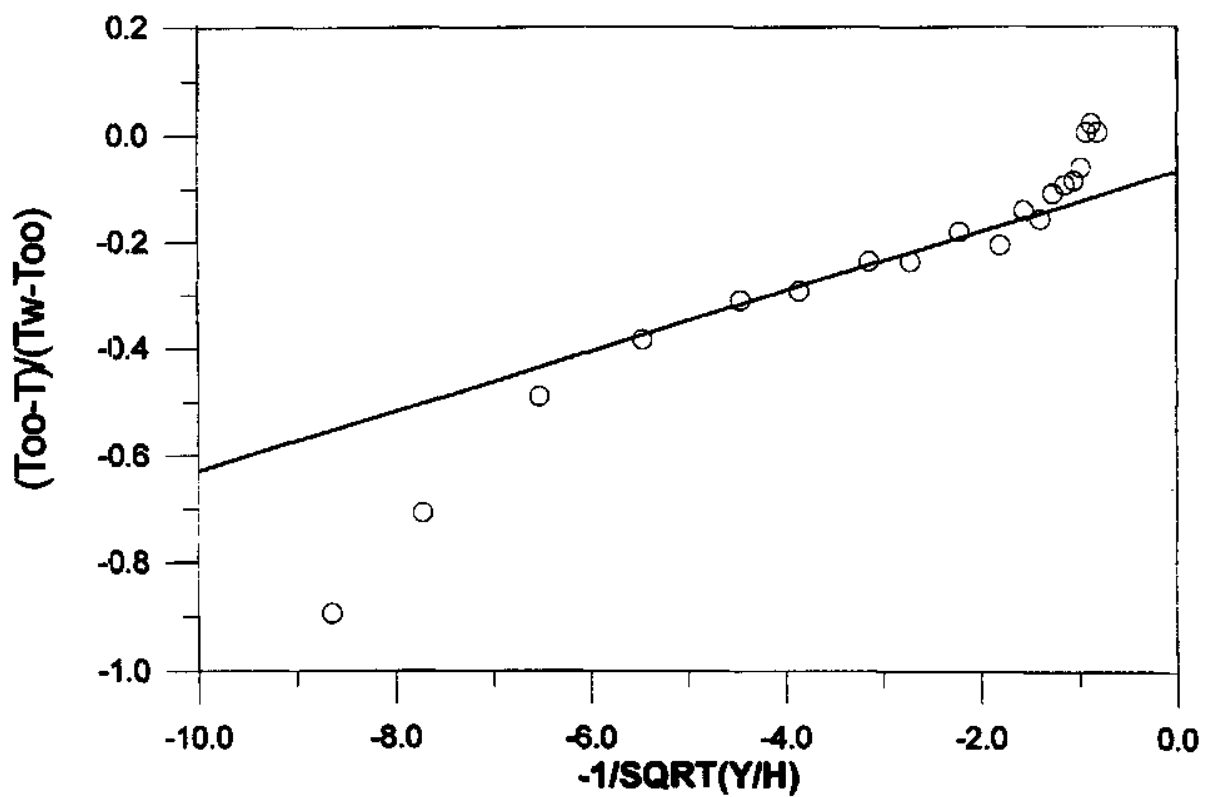

Fig. 4. Temperature profile at separation according to Vogel and Eaton [10]. $x^{*}=0.05$.

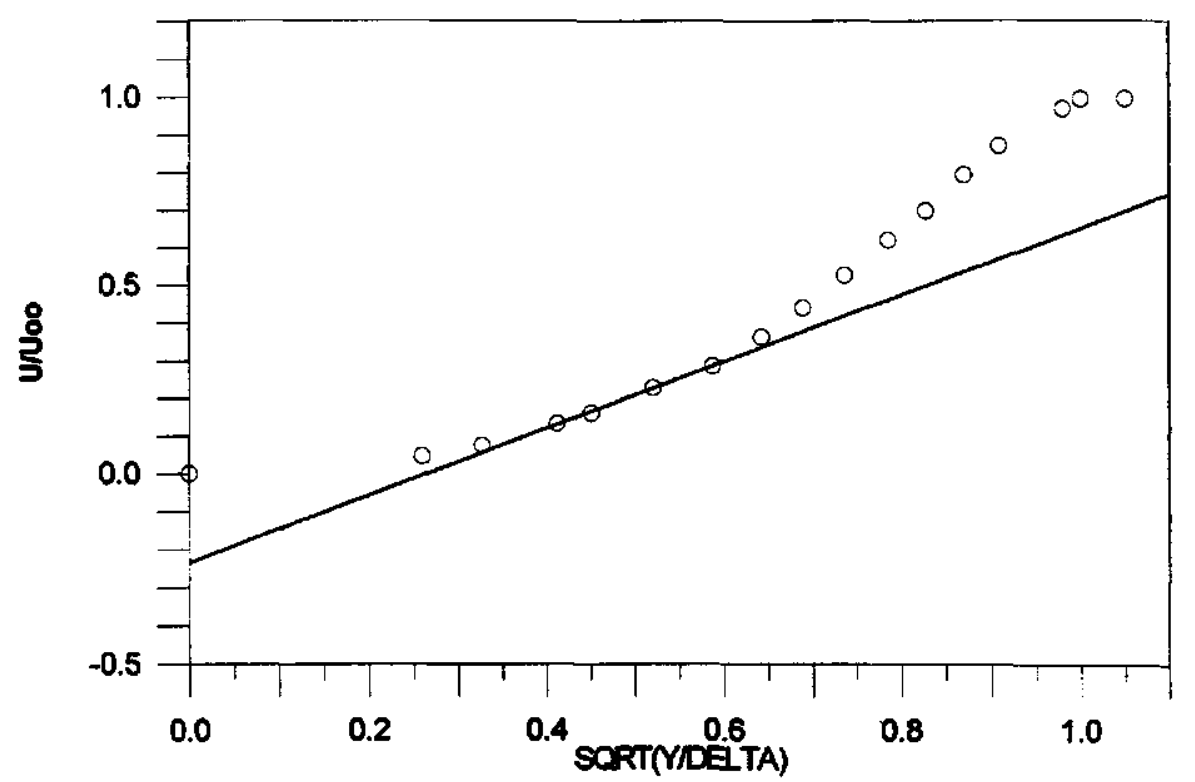

Fig. 5. Velocity profile at separation according to Dengel and Fernholtz [27]. $C_{\mathrm{f}}=0.0, \delta=71.87 \mathrm{~mm}$, $U_{\infty}=9.211 \mathrm{~m} \mathrm{~s}^{-1}$.

$$
\tau_{\mathrm{w}}=\kappa c_{\mu}^{1 / 4} \frac{\rho \sqrt{K_{\mathrm{p}}}}{\ln E_{2} y_{2}} u_{\mathrm{p}} .
$$

In the above equation, $u_{\mathrm{p}}$ denotes the flow velocity at the first grid point,

$$
\begin{gathered}
y_{1}=y_{\mathrm{p}} c_{\mu}^{1 / 4} \rho \sqrt{K_{\mathrm{p}}} / v \\
y_{2}=y_{\mathrm{p}} / L
\end{gathered}
$$

$$
L=\frac{\frac{-\tau_{\mathrm{w}}}{\rho} \pm\left|\frac{\tau_{\mathrm{w}}}{\rho}\right| \sqrt{1+\left(\frac{\rho}{\tau_{\mathrm{w}}}\right)^{2} \nu \frac{1}{\rho} \frac{\mathrm{d} \bar{P}_{\mathrm{w}}}{\mathrm{d} x} 2 u_{\tau}}}{\frac{1 \mathrm{~d} \bar{P}_{\mathrm{w}}}{\rho} \mathrm{d} x}
$$$$
u_{\imath}=c_{\mu}^{1 / 4} \rho \sqrt{K_{p}}
$$ 


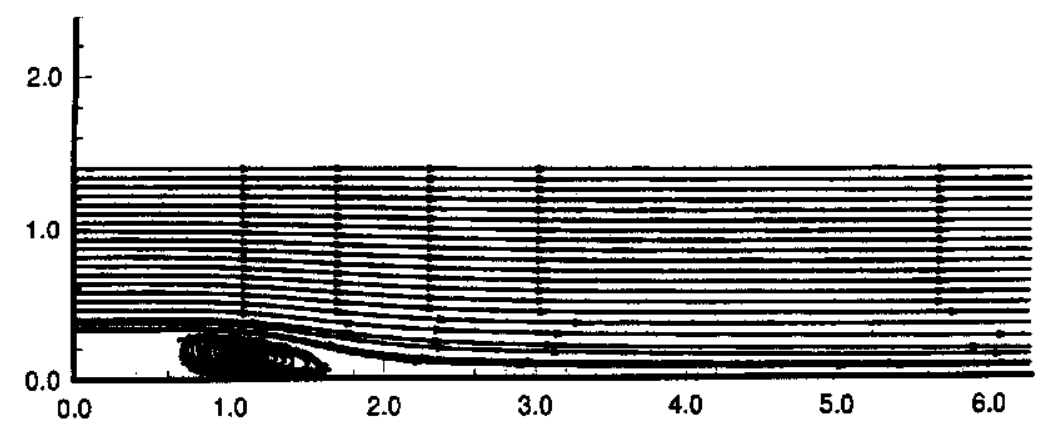

Fig. 6. Flow streamlines for the Vogel and Eaton [10] geometry. The step height is $0.379 \mathrm{dm}$.

(in $\left.E_{1}\right) / k=5$ and $\left(\ln E_{2}\right) / k=10.8$. For the evaluation of the temperature profile, the boundary condition at the wall was taken as

$$
\begin{aligned}
& \frac{\left(H_{\mathrm{p}}-H_{\mathrm{w}}\right) \rho c_{\mu}^{1 / 4} K_{\mathrm{p}}^{1 / 2}}{Q_{\mathrm{w}}} \\
& \quad=\frac{P r_{t}}{\kappa} \ln \left|\frac{\sqrt{\tau_{\mathrm{w}} / \rho+\frac{\mathrm{d} P_{\mathrm{w}}}{\mathrm{d} x} y_{2}}-\sqrt{\tau_{\mathrm{w}} / \rho}}{\sqrt{\tau_{\mathrm{w}} / \rho+\frac{\mathrm{d} P_{\mathrm{w}}}{\mathrm{d} x} y_{2}}+\sqrt{\tau_{\mathrm{w}} / \rho}}\right|+C_{t}+C_{q}
\end{aligned}
$$

where $H_{\mathrm{p}}$ and $K_{\mathrm{p}}$ stand for the enthalpy and the turbulent kinetic energy at the first grid point. $Q_{\mathrm{w}}$ is the heat flux at the wall and $C_{q}$ is given by

$$
\begin{array}{ll}
C_{q}=12.5 \operatorname{Pr}^{2 / 3}+2.12 \ln P r-5.3, & \text { if } P r>0.5 \\
C_{q}=12.5 P^{2 / 3}+2.12 \ln P r-1.5, & \text { if } P r \leqslant 0.5 .
\end{array}
$$

The concept of a turbulent Prandtl number was used here. The calculated streamlines are shown in Fig. 6. The calculated reattachment point is located at 5-7/10 step heights downstream of separation as opposed to the $6-2 / 3$ step heights from the experiments. Figure 7 shows the calculated velocity profiles

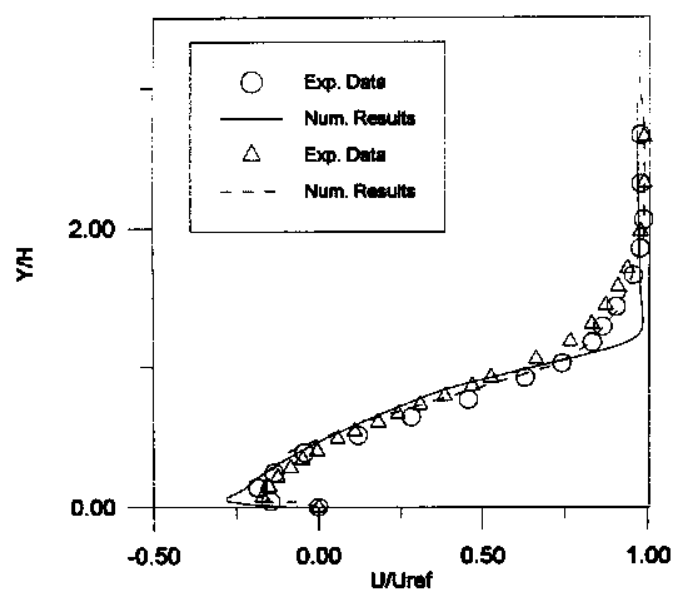

Fjg. 7. Calculated velocity profiles for stations $x^{*}=-0.32$ $\left(\triangle, U_{\infty}=10.33 \mathrm{~m} \mathrm{~s}^{-1}\right)$ and $x^{*}=-0.44\left(O, U_{\infty}=10.46 \mathrm{~m}\right.$ $\left.\mathrm{s}^{-1}\right)$. Data of Vogel and Eaton [10]. for several stations upstream of the separation. As can be seen, the theoretical predictions are very good.

The calculated values of the skin-friction for the backward-facing step flow geometry of Vogel and Eaton [10] are shown in Fig. 8. The improvement in the predictions with the introduction of the new law of the wall formulation is remarkable. The assessment of the point of minimum $C_{\mathrm{f}}$ value is, in particular, very good. The next figure, Fig. 9, shows the skinfriction predictions for the divergent channel flow geometry of Driver and Seegmiller [11]. In this figure a comparison is also made with a RSM simulation of the flow by Hwang and Peng [12]. The results obtained with the present formulation are clearly comparable with the more sophisticated RSM formulation. The Stanton number predictions are shown in Fig. 10 for the backward-facing step flow geometry. The results are reasonable. Possibly, the lack of a better agreement is the result of the turbulent Prandtl number formulation.

\section{FINAL REMARKS}

The present analysis has shown through the concept of $\eta$-limit of Kaplun how the two-deck turbulent boundary layer structure develops into a one-deck structure near a separation point. This result seems, at first, contradictory to the three-layer structure found by other authors (Melnik [4], Durbin and Belcher [6]). However, we point out that all local equations derived by these authors are intermediate equations, in the sense of Kaplun, being therefore, contained in the domain of validity of the principal equations here derived. In other words we may say that those theories are 'contained' in the present theory. Of course, the principal equations are of difficult solution, do not provide closed analytical solutions; however, only these equations give fundamental insight to understand how the viscous and defect layers merge as a separation point is approached.

For the temperature boundary layer, the three limit cases $\operatorname{ord}(P r)<\operatorname{ord}(1)$, ord $(P r)=\operatorname{ord}(1)$ and ord$(P r)>\operatorname{ord}(1)$ were studied and law of the wall expressions were derived. 


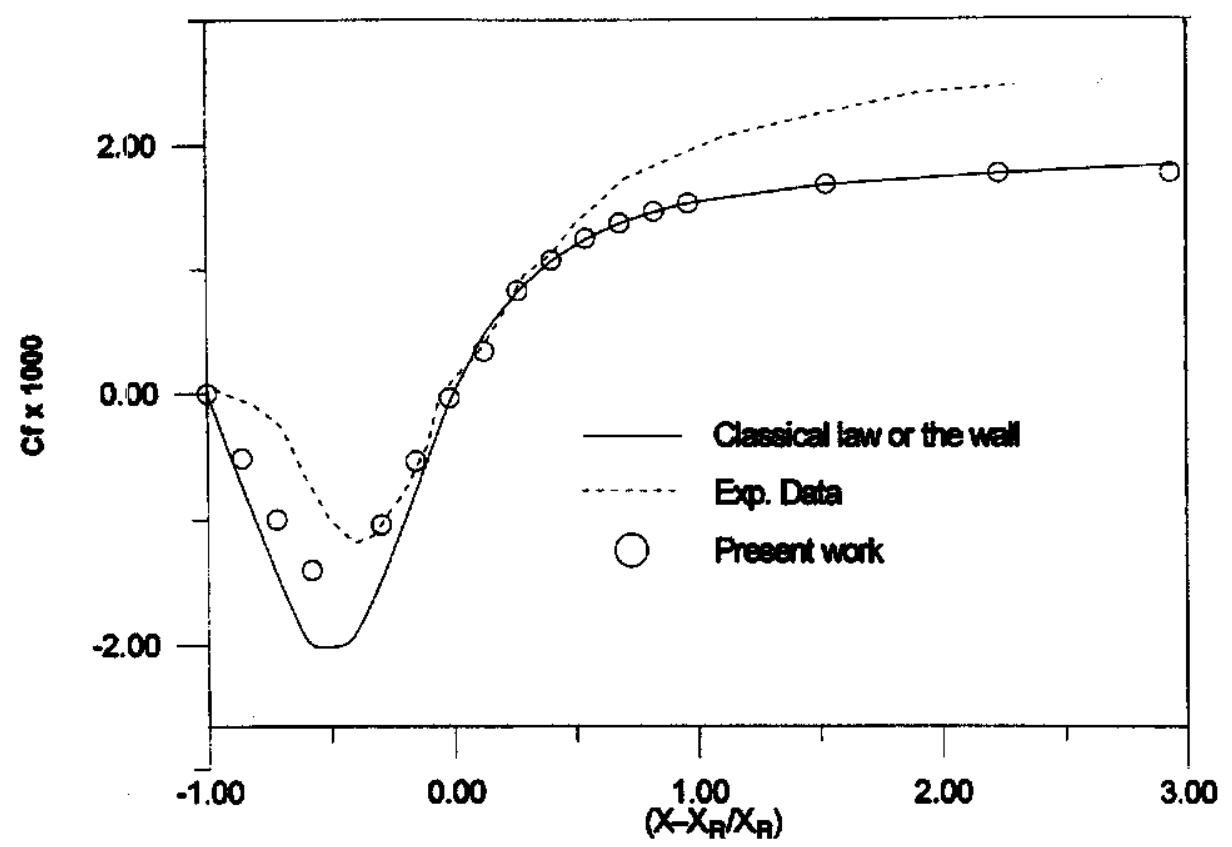

Fig. 8. Skin-friction results for the backward-facing step flow geometry of Vogel and Eaton [10].

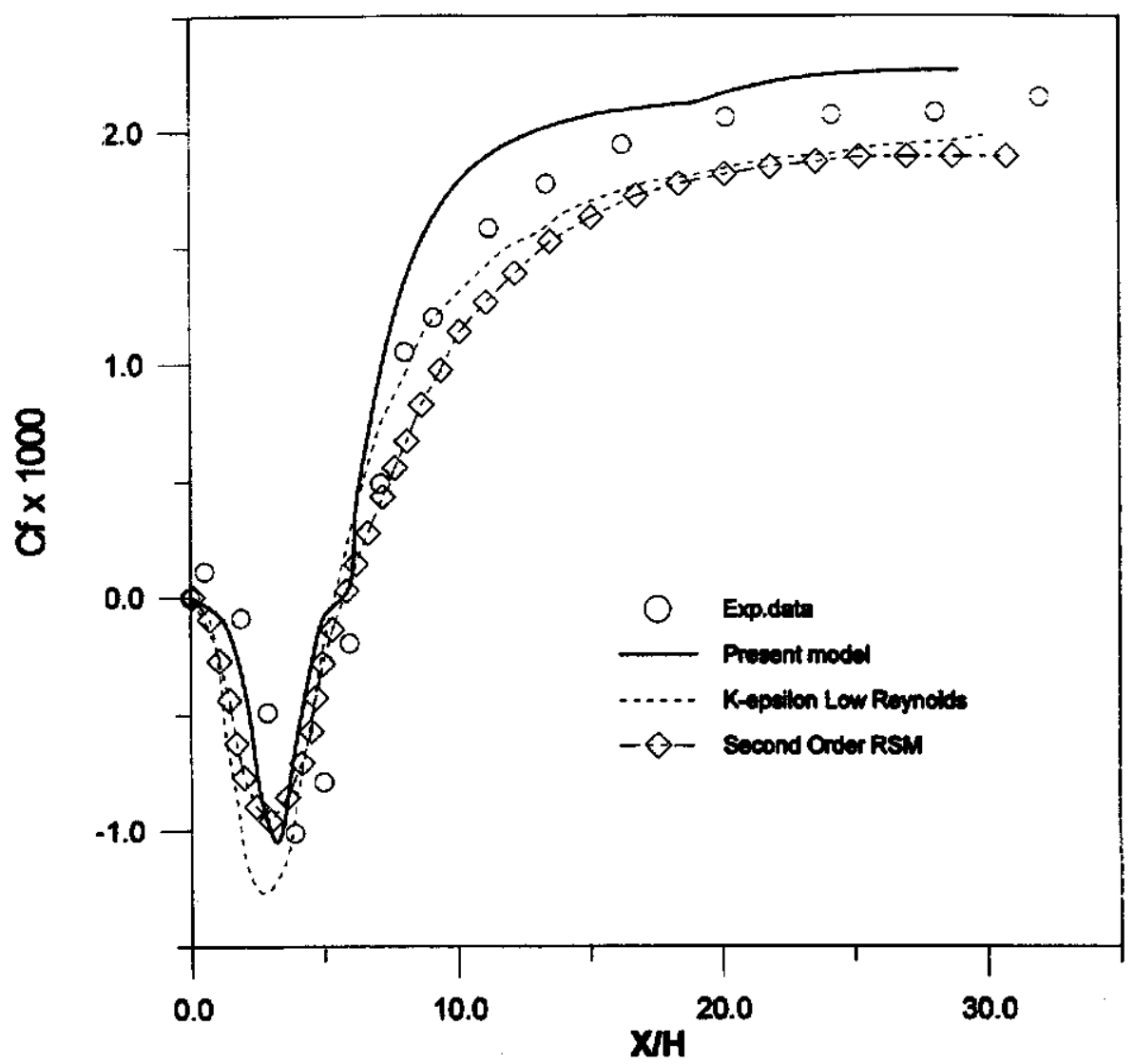

Fig. 9. Skin-friction results for the divergent channel flow geometry of Driver and Seegmiller [11]. 


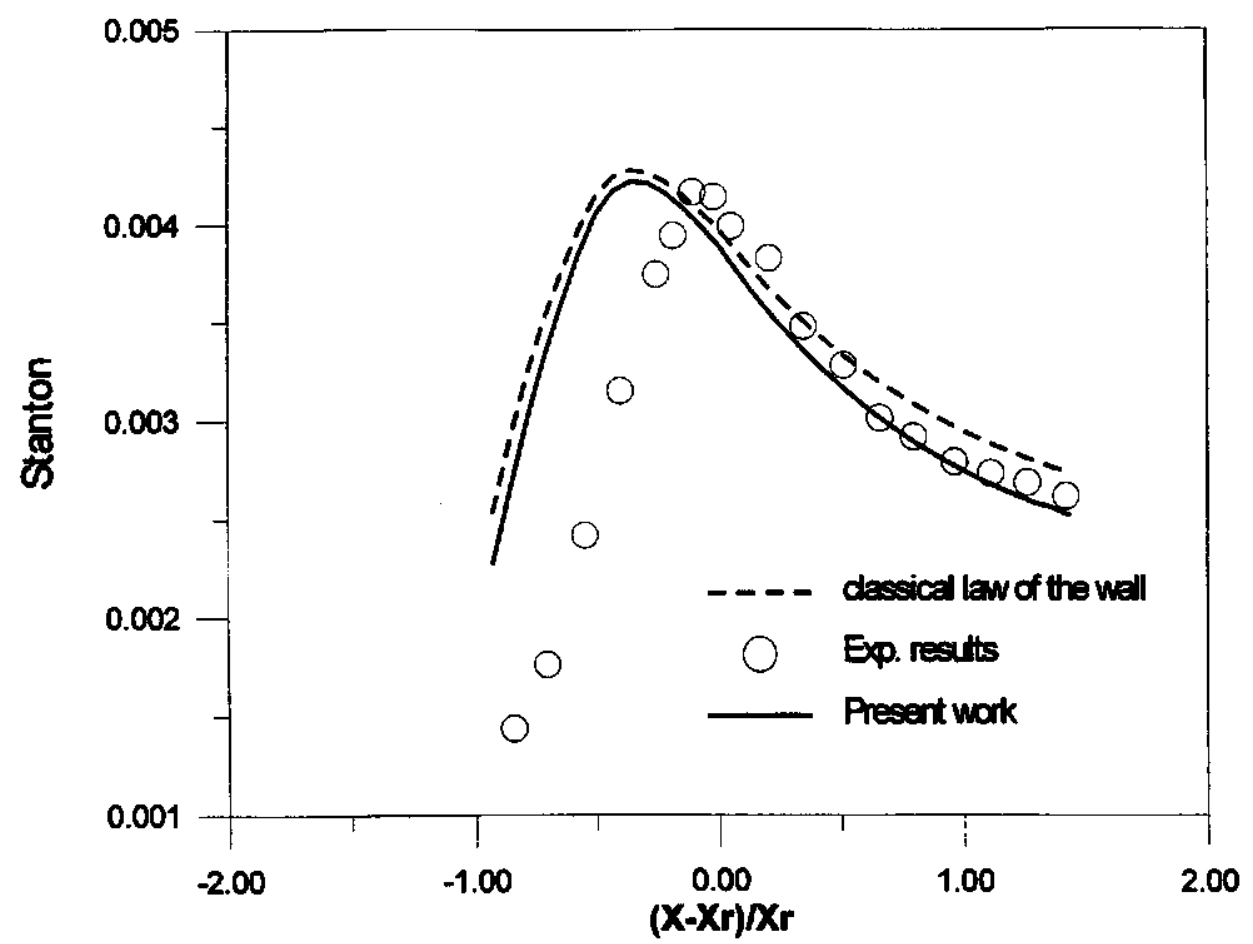

Fig. 10. Stanton number predictions for the backward-facing flow geometry of Vogel and Eaton [10].

An important assumption of this work is that the reference velocity can be defined through an algebraic equation. The resulting behaviour of $u_{R}$ accommodates the boundary layer structure, and gives values of $u_{\imath}$, which are very much in accordance with the data in literature.

Finally, a comparison of the present theory with the data of Vogel and Eaton [10], and of Driver and Seegmiller [11] shows that the present hypotheses are plausible, leading to consistent results. The new concepts here derived should therefore instigate new thoughts on separating turbulent boundary layers, in particular, on the nature of its asymptotic structure.

Acknowledgements-The present work was financially supported by the Brazilian National Research Council (CNPq) through Grant no. 350183/93-7. One of the authors, D.O.A.C., is grateful to the Brazilian National Research Council (CNPq) for the award of a scholarship during the course of the research.

\section{REFERENCES}

1. Yajnik, K. S., Asymptotic theory of turbulent shear flow. Journal of Fluid Mechanics, 1970, 42, 41 1-427.

2. Mellor, G. L., The large Reynolds number asymptotic theory of turbulent boundary layers. International Journal of Engineering Science, 1972, 10, 851-873.

3. Bush, W. B. and Fendell, F. E., Asymptotic analysis of turbulent channel and boundary layer flow. Journal of Fluid Mechanics, 1972, 56, 657-681.

4. Melnik, R. E., An asymptotic theory of turbulent separation. Compt and Fluids, 1989, 17, 165-184.

5. Sychev, V. V. and Sychev, V. V., On turbulent boundary layer structure. P.M.M. U.S.S.R., 1987, 51, 462-467.

6. Durbin, P. A. and Belcher, S. E., Scaling of adverse- pressure-gradient turbulent boundary layers. Journal of Fluid Mechanics, 1992, 238, 699-722.

7. Mellor, G. L., The effects of pressure gradients on turbulent flow near a smooth wall. Journal of Fluid Mechanics, 1966, 24, 255-274.

8. Gersten, K., Some contributions to asymptotic theory for turbulent flows. 2nd International Symposium on Transport Phenomena in Turbulent Flows. Tokyo, Japan, 1987, pp. 201-214.

9. Kaplun, S., Fluid Mechanics and Singular Perturbations. Academic Press, 1967.

10. Vogel, J. C. and Eaton, J. K., Combined heat transfer and fluid dynamic measurements downstream of a backward facing step. Journal of Heat Transfer, 1985, 107, 922-929.

11. Driver, D. M. and Seegmiller, H. L., Features of a reattaching turbulent shear layer in divergent channel flow. AIAA Journal, 1995, 23, 163-171.

12. Hwang, R. R. and Peng, Y. F., Computation of backward-facing step flows by a second-order Reynolds stress closure model. International Journal for Numerical Methods in Fluids, 1995, 21, 223-235.

13. Van Dyke, M., Perturbation Methods in Fluid Mechanics. Parabolic Press, 1972.

14. Lagerstrom, P. A. and Casten, R. G., Basic concepts underlying singular perturbation techniques. SIAM Review, 1972, 14, 63-120.

15. Meyer, R. E., On the approximation of double limits by single limits and the Kaplun extension theorem. I. Inst. Maths. Applics., 1967, 3, 245-249.

16. Freund, D. D., A note on Kaplun limits and double asymptotics. Proc. American Math. Soc., 1972, 35, 464 270.

17. Silva Freire, A. P. and Hirata, M. H., Approximate solutions to singular perturbation problems: the intermediate variable technique. J. Math. Analysis and Appl., $1990,145,241-253$.

18. Orlando, A. F., Moffat, R. J. and Kays, W. M., Heat transfer in turbukent flows under mild and strong adverse 
pressure gradient conditions for an arbitrary variation of the wall temperatures. Report HMT-17, Stanford University, 1974.

19. Kistler, A. L., Fluctuation measurements in a supersonic turbulent boundary layer. Physics of Fluids, 1959, 2, 290296.

20. Kistler, A. L. and Chew, W. S., A fluctuating pressure field in a turbulent boundary layer. Journal of Fluid Mechanics, 1963, 16, 41-64.

21. Cruz, D. O, A. and Silva Freire, A. P., The asymptotic structure of the thermal turbulent boundary layer near a separation point. In Turbulence, Heat and Mass Transfer, ed. K. Hanjalic. Begell House Inc. Publishers, 1995.

22. Stratford, B. S., An experimental flow with zero skinfriction throughout its region of pressure rise. Journal of Fluid Mechanics, 1959, 5, 17-35.

23. Townsend, A. A., The Structure of Turbulent Shear Flow. Cambridge University Press, 1976.

24. Landau, L. D. and Lifschitz, E. M., Fluid Mechanics. Academic Press, 1987.

25. Kader, B. A. and Yaglom, A. M., Similarity treatment of moving-equilibrium turbulent boundary layers in adverse pressure gradients. Journal of Fluid Mechanics, $1979,89,305-342$.

26. Simpson, R. L., Chew, Y. T and Schivaprasad, B. G., The structure of a separating boundary layer. Part 1 : Mean flow and Reynolds stresses. Journal of Fluid Mechanics, 1981, 113, 23-51.

27. Thompson, B. E. and Whitelaw, J. H., Characteristics of trailing edge flow with turbulent boundary layer separation. Journal of Fluid Mechanics, 1985, 157, 305-326.

28. Dengel, P. and Fernholtz, H. H., An experimental investigation of an incompressible turbulent boundary layer in the vicinity of separation. Journal of Fluid Mechanics, $1990,22,615-636$.

29. Peric, M. and Scheuerer, G., CAST-a finite volume method for predicting two-dimensional flow and heat transfer phenomena, GRS-Technische Notiz Srr-89-01, 1989.

30. Launder, B. E. and Spalding, D. B., The numerical computation of turbulent flows. Computer Methods in Applied Mechanics, 1974, 3, 269-289. 